Eur J Cancer. 2010 May ; 46(7): 1177-1180. doi:10.1016/j.ejca.2010.02.039.

\title{
Cancer metastasis as a therapeutic target
}

\author{
Jonathan Sleeman ${ }^{a, b}$ and Patricia S. Steeg ${ }^{c, *}$ \\ aUniversitätsmedizin Mannheim, University of Heidelberg, Ludolf-Krehl-Str. 13-17, D-68167 \\ Mannheim, Germany \\ bKarlsruher Institut für Technologie (KIT), Institut für Toxikologie und Genetik ITG, Postfach 3640, \\ D-76021 Karlsruhe, Germany \\ 'Women's Cancers Section, Laboratory of Molecular Pharmacology, Center for Cancer Research, \\ National Cancer Institute, Bethesda, MD, United States
}

\begin{abstract}
Despite many years of basic and clinical research aimed at curbing tumour growth, metastasis remains the prime reason why cancer patients succumb to their disease. Effective translational research is urgently required, yet is not always easy to achieve. Here we review reasons why metastasis as a disease process has proven difficult to control, and suggest ways in which translational research in this area can be strengthened and advanced.
\end{abstract}

\section{Keywords}

Cancer; Metastasis; Translational research

Cancer is recognised worldwide to be a major health problem. Within Europe there are an estimated 3.2 million new cases and 1.7 million deaths each year. ${ }^{1}$ Of these deaths, it is estimated that more than $90 \%$ are due to the direct or indirect effects of metastases. ${ }^{2}$ Patient prognosis is therefore intimately connected with metastatic disease, as reflected in the staging systems for many types of cancer. The diagnosis 'metastatic cancer' is considered terminal for most cancer types. The quality of life consequences of living with metastatic cancer are being increasingly recognised by patient groups, with fear of the ' $\mathrm{M}$ ' word superseding that of the ' $\mathrm{C}$ ' word. Metastatic disease, therefore, represents a major public health problem, affecting cancer patients and their families, as well as health care systems and the broader economy. Despite this, progress in developing treatments for metastatic disease remains slow. In this article we survey the reasons why this might be the case and suggest ways in which translational metastasis research can be promoted.

Treatments for metastatic cancer are currently similar to those used for primary tumours. Radiation therapy is a mainstay of treatment, and patients receive multiple courses of chemotherapy depending on the cancer histology and toxicity profile. Surgery is rarely

\footnotetext{
"Corresponding author: Tel.: +1 301496 9753; fax: +1 301402 8910., steegp@ mail.nih.gov (P.S. Steeg). 
performed on metastatic lesions. More recently, biological/molecular targeted therapies have come increasingly to the fore. The underlying assumption has been that metastases were essentially similar to primary tumours and that tumour growth control should effectively suppress metastatic growth. This assumption is being challenged by accumulating molecular evidence and pre-clinical modelling. ${ }^{3,4}$

Tumour growth control in itself can actually promote rather than suppress the formation and growth of metastases, and there are increasing numbers of examples where chemotherapy, radiotherapy and biological/targeted therapies have this effect. For example, adjuvant radiotherapy used for local growth control can promote metastasis via the so-called tumour bed effect, in which tumour recurrence in the irradiated field is associated with higher metastasis and poor prognosis. ${ }^{5}$ The underlying reasons for this effect remain unclear, but hypoxia and/or the upregulation of metastasis promoting factors in the irradiated tumour bed may be involved. ${ }^{6}$ As another example, anti-angiogenesis therapy showed great promise in pre-clinical studies as a means of controlling tumour growth, yet clinical trials of these therapies showed surprisingly small effects on patient survival. ${ }^{7-10}$ Recent stud ies indicate that anti-angiogenesis therapies can promote experimental metastasis, ${ }^{11,12}$ possibly by increasing hypoxia, providing a mechanism to explain the discrepancy between pre-clinical studies and the clinical trials. In view of these and other observations we have suggested that novel cancer therapies should investigate possible effects on metastasis during pre-clinical development. ${ }^{13}$

Although treatment of metastases often assumes they are similar to primary tumours, there are many reasons to believe that this is not the case. For example, metastases can exhibit substantial differences in gene expression patterns compared with primary tumours ${ }^{3,4}$ and obviously grow within different organ microenvironments. By understanding and capitalising on these differences, it may be possible to develop more effective treatments. The recognition, for example, that metastatic tumour cells in the bone interact with osteoblasts and osteoclasts, leading to metastatic growth and disturbance of bone homeostasis in a vicious positive feed-back loop, prompted the use of bisphosphonates to control bone metastases. ${ }^{14,15}$ Bisphosphonates act on the osteoclast population, promoting their apoptosis and suppressing their activity and formation. The use of bisphosphonates has proven highly effective in controlling the osteoblastic and osteolytic changes associated with bone metastases. Investigators working with RANK-L inhibitors, a molecularly targeted therapy designed to interrupt the vicious cycle, have just reported encouraging phase III clinical trial results. ${ }^{14,15}$

To facilitate the rational design of anti-metastasis therapies, the process of metastasis needs to be understood. In recent years it has become clear that there are many aspects of this process that we do not understand. The prevailing paradigm of a stochastic selection-based evolution of metastatic progression driven by cumulative genetic aberrations late in the disease does not seem to tell the whole story. ${ }^{16}$ There is evidence for a hierarchical organisation within tumour cell populations underpinned by cancer stem cells, the only cells in the population able to initiate the formation of a new tumour. These cancer stem cells would be expected to contribute decisively to metastasis formation. ${ }^{17}$ Evidence from gene expression profiling of primary tumours allows the identification of gene expression profiles 
that predict metastatic potential, suggesting that metastatic propensity is determined early during tumourigenesis. ${ }^{18}$ Genetic analyses of disseminated tumours cells, as well as the analysis of animal models, suggest that dissemination of tumour cells can be a very early event, even at a premalignant stage. ${ }^{19}$ There is also increasing appreciation of the role that the organ microenvironment makes to determining whether or not metastases form, including the formation of niche structures that support metastatic growth and are possibly predetermined by primary tumours before metastases seed and grow. ${ }^{20}$ The regulation of dormancy and organ-specific metastasis remains poorly understood. ${ }^{21}$ The mobilisation and recruitment of bone marrow-derived cells are also emerging as important echanisms in the development of metastases. ${ }^{22}$ Together, this short summary of recent observations serves to show how much more research is required to understand the mechanism and process of metastasis, a pre-requisite for effective rational design of novel therapies. Put another way, in order to carry out translational research, you need to have something reliable to translate. A close interaction between clinicians and basic researchers is required to understand mechanistically the process of metastasis in patients, and the problems associated with treating metastatic disease. In this regard it is important to note that translational research is not a one-way street from bed to bedside: observations made during the clinical treatment of metastatic disease need to feed into basic research efforts.

Clinical trials often do not address directly effects on metastasis. Because of its nature, assessment of the formation and growth of metastases is of necessity a long-term undertaking, making inclusion of metastasis as an end-point in clinical trials often prohibitively expensive. Nevertheless, the end-points in clinical trials determine their utility and the lack of direct assessment of the effects on metastasis is therefore a major bottleneck in the development of effective metastasis therapies. The amount of potentially useful clinical information lost is staggering: information on sites of new metastases could support the development and evaluation of site-specific therapies. In adjuvant trials, information on initial metastatic relapse, as well as subsequent sites of relapse, would be constructive.

To make clinical trials that address metastasis formation economically viable, surrogate assays that give an inexpensive and early readout of possible effects on metastasis would be desirable. For example, assessment of blood-borne tumour cells or proteins released from metastases may be useful for monitoring effects of drugs on metastasis in a minimally invasive and cost-effective manner. ${ }^{23}$ Improved imaging techniques that are currently in preclinical development and that offer improved temporal and spatial resolution may also be useful in this regard. For example, flat panel volume computed tomography offers the possibility of effectively imaging relatively small blood vessels within tumours and metastases, permitting early assessment of the effects of drugs aimed at metastases on both the metastases themselves and their blood vessels. ${ }^{24}$ Proteomic analysis of blood and improved imaging also hold the promise of allowing the development of metastases to be detected at an early stage, potentially improving the efficacy of therapy by treating metastases while they are still small.

Aside from surrogate end-points, consideration should be given to other measures of patient benefit. Most metastasis-directed compounds reported to date interrupt the metastatic process and therefore prevent it. However, in early clinical testing these agents are given to 
patients with widespread, incurable metastatic disease and are asked to shrink a metastatic deposit, a completely different situation. While the optimal trial design to prevent metastasis is conducted in the adjuvant setting, these are often long, expensive trials and are only conducted after promising Phase I and II studies. Newer trial designs to assess progressionfree survival in the early metastatic setting may be optimal, with the development of a new metastasis, and not shrinkage of the initial lesion as the primary endpoint. This field will require clinicians and scientists to go into business together to work out these important but difficult issues.

Given the clinical importance of metastasis for cancer patients, the limited treatment options for metastatic disease and the open question of how metastasis works, how much research funding is being directed at the problem? What proportion of funding for cancer research ends up focused on metastases? Reliable figures are difficult to come by. Given the fundamental integration of the process of metastasis in tumour development and growth, it is sometimes difficult to delineate accurately the percentage of funding devoted to metastasis research. Nevertheless, by analysing the reports from a number of international governments and organisations concerning their funding for cancer research and scanning for key words such as metastasis, dissemination, progression and invasion, we have tried to determine the amount of cancer research funding devoted to basic and clinical studies on metastasis (Table 1). Although there is considerable variation, the median spent on metastasis research is around $5 \%$ of total cancer research funding. Is this sufficient? The answer is difficult to answer objectively, as many different factors of necessity need to be balanced, all of which are relevant to patient treatment. Nevertheless, given the central importance of metastasis to the prognosis and outcome of cancer patients, it may be argued that in many countries more funding should be directed toward metastasis research.

In summary, combating metastasis formation and growth is the key to successfully treating cancer. Traditional growth control approaches are inadequate and can even be detrimental in the long term: new therapies built upon a solid understanding of the process of metastatic disease are urgently required. In turn, this demands an increased pre-clinical knowledge base that capitalises on major conceptual advances made in recent years, as well as a rational approach to the design of clinical trials with the inclusion of metastasis as an end-point. Together, these observations speak for the necessity of increasingly close interactions between basic and clinical scientists, as well as the enhanced levels of research funding required to alleviate this major clinical problem.

\section{Acknowledgements}

J.P.S. gratefully acknowledges funding from the European Union under the auspices of the FP7 collaborative project TuMIC, Contract No. HEALTH-F2-2008-201662. P.S.S. is supported by the Intramural Program of the National Cancer Institute, USA.

\section{REFERENCES}

1. Ferlay J, Autier P, Boniol M, et al. Estimates of the cancer incidence and mortality in Europe in 2006. Ann Oncol 2007;18(3):581-92. [PubMed: 17287242]

2. Sporn MB. The war on cancer. Lancet 1996;347(9012):1377-81. [PubMed: 8637346] 
3. Steeg PS, Theodorescu D. Metastasis: a therapeutic target for cancer. Nat Clin Pract Oncol 2008;5(4):206-19. [PubMed: 18253104]

4. Steeg PS. Heterogeneity of drug target expression among metastatic lesions: lessons from a breast cancer autopsy program. Clin Cancer Res 2008;14(12):3643-5. [PubMed: 18559575]

5. Suit HD. Local control and patient survival. Int J Radiat Oncol Biol Phys 1992;23(3):653-60. [PubMed: 1612967]

6. Rofstad EK, Mathiesen B, Henriksen K, Kindem K, Galappathi K. The tumor bed effect: increased metastatic dissemination from hypoxia-induced up-regulation of metastasis-promoting gene products. Cancer Res 2005;65(6):2387-96. [PubMed: 15781654]

7. Hurwitz H Integrating the anti-VEGF-A humanized monoclonal antibody bevacizumab with chemotherapy in advanced colorectal cancer. Clin Colorectal Cancer 2004; 4(Suppl 2):S62-8. [PubMed: 15479481]

8. Sandler A, Gray R, Perry MC, et al. Paclitaxel-carboplatin alone or with bevacizumab for nonsmall-cell lung cancer. New Engl J Med 2006;355(24):2542-50. [PubMed: 17167137]

9. Escudier B, Pluzanska A, Koralewski P, et al. Bevacizumab plus interferon alfa-2a for treatment of metastatic renal cell carcinoma: a randomised, double-blind phase III trial. Lancet 2007;370(9605): 2103-11. [PubMed: 18156031]

10. Miller K, Wang M, Gralow J, et al. Paclitaxel plus bevacizumab versus paclitaxel alone for metastatic breast cancer. New Engl J Med 2007;357(26):2666-76. [PubMed: 18160686]

11. Ebos JM, Lee CR, Cruz-Munoz W, et al. Accelerated metastasis after short-term treatment with a potent inhibitor of tumor angiogenesis. Cancer Cell 2009;15(3):232-9. [PubMed: 19249681]

12. Paez-Ribes M, Allen E, Hudock J, et al. Antiangiogenic therapy elicits malignant progression of tumors to increased local invasion and distant metastasis. Cancer Cell 2009;15(3): 220-31. [PubMed: 19249680]

13. Steeg PS, Anderson RL, Bar-Eli M, et al. Preclinical drug development must consider the impact in metastasis. Clin Cancer Res 2009;15(14):4529-30. [PubMed: 25278743]

14. Steeg PS. Tumor metastasis: mechanistic insights and clinical challenges. Nat Med 2006;12(8): 895-904. [PubMed: 16892035]

15. Neville-Webbe HL, Coleman RE. Bisphosphonates for the treatment and prevention of metastatic bone disease. Eur J Cancer 2010;46(7):1211-22. [PubMed: 20347292]

16. Sleeman JP, Cremers N. New concepts in breast cancer metastasis: tumor initiating cells and the microenvironment. Clin Exp Metastasis 2007;24(8):707-15. [PubMed: 17972145]

17. Monteiro J, Fodde R. Cancer stemness and metastasis: therapeutic consequences and perspectives. Eur J Cancer 2010;46(7):1198-203. [PubMed: 20303259]

18. Bernards R, Weinberg RA. A progression puzzle. Nature 2002;418(6900):823. [PubMed: 12192390]

19. Klein CA. Parallel progression of primary tumours and metastases. Nat Rev Cancer 2009;9(4): 302-12. [PubMed: 19308069]

20. Psaila B, Lyden D. The metastatic niche: adapting the foreign soil. Nat Rev Cancer 2009;9(4):28593. [PubMed: 19308068]

21. Barkan D, Green JE, Chambers AF. Extracellular matrix: a gatekeeper in the transition from dormancy to metastatic growth. Eur J Cancer 2010;46(7):1181-8. [PubMed: 20304630]

22. Alison MR, Lim S, Houghton JM. Bone marrow-derived cells and epithelial tumours: more than just an inflammatory relationship. Curr Opin Oncol 2009;21(1):77-82. [PubMed: 19125022]

23. Brouwers FM, Petricoin EF 3rd, Ksinantova L, et al. Low molecular weight proteomic information distinguishes metastatic from benign pheochromocytoma. Endocr Relat Cancer 2005;12(2):26372. [PubMed: 15947101]

24. Missbach-Guentner J, Dullin C, Zientkowska M, et al. Flat-panel detector-based volume computed tomography: a novel 3D imaging technique to monitor osteolytic bone lesions in a mouse tumor metastasis model. Neoplasia 2007;9(9):755-65. [PubMed: 17898871] 


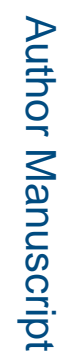

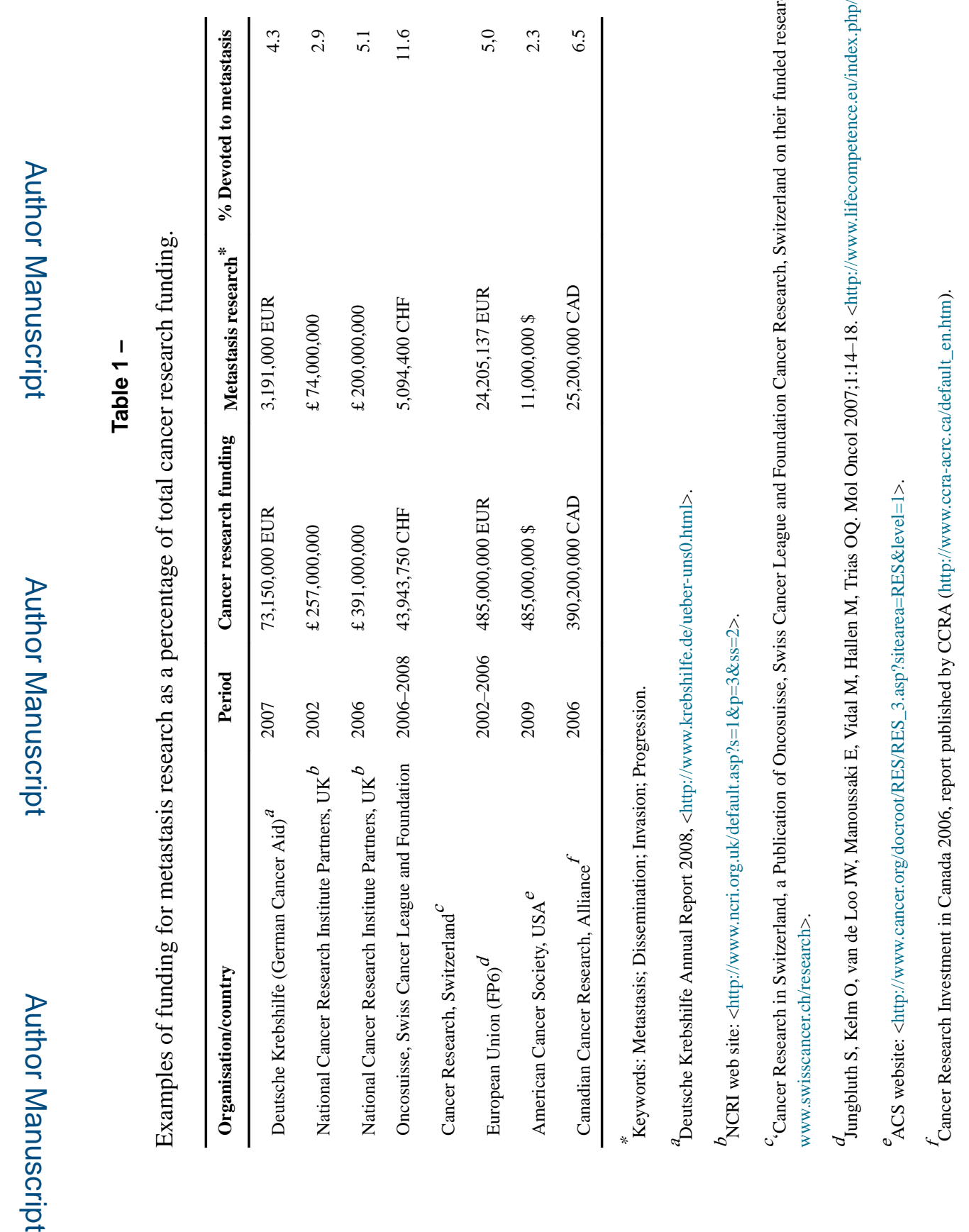

Eur J Cancer: Author manuscript; available in PMC 2019 June 03. 